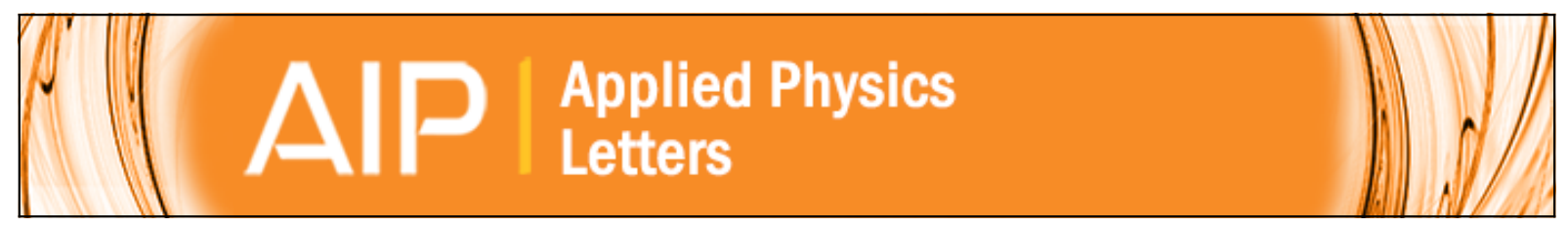

\title{
Metal-slotted polymer optical waveguide device
}

Seong-Ku Kim, Yu-Chueh Hung, W. Yuan, DongHo Cha, K. Geary, H. R. Fetterman, Soon Mok Ha, Qibing Pei, Jindong Luo, XingHua Zhou, Alex K.-Y. Jen, M.-S. Kwon, and W. H. Steier

Citation: Applied Physics Letters 90, 243507 (2007); doi: 10.1063/1.2746414

View online: http://dx.doi.org/10.1063/1.2746414

View Table of Contents: http://scitation.aip.org/content/aip/journal/apl/90/24?ver=pdfcov

Published by the AIP Publishing

\section{Articles you may be interested in}

Long-range surface plasmon-polariton waveguides and devices in lithium niobate

J. Appl. Phys. 101, 113114 (2007); 10.1063/1.2739300

Side-chain electro-optic polymer modulator with wide thermal stability ranging from $-46^{\circ} \mathrm{C}$ to $95^{\circ} \mathrm{C}$ for fiberoptic gyroscope applications

Appl. Phys. Lett. 87, 061112 (2005); 10.1063/1.2009807

Electro-optic phase modulator using metal-defined polymer optical waveguide

Appl. Phys. Lett. 87, 011107 (2005); 10.1063/1.1991998

Flexible low-voltage electro-optic polymer modulators

Appl. Phys. Lett. 82, 4432 (2003); 10.1063/1.1586474

Dual-functional polymeric waveguide with optical amplification and electro-optic modulation

Appl. Phys. Lett. 72, 2806 (1998); 10.1063/1.121464

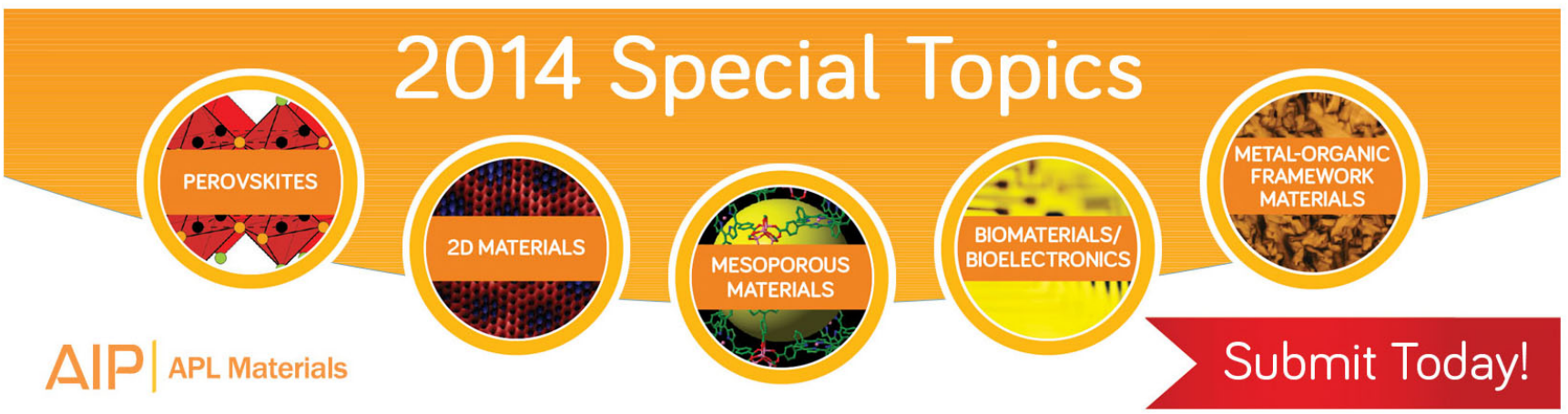




\title{
Metal-slotted polymer optical waveguide device
}

\author{
Seong-Ku Kim, ${ }^{\text {a) }}$ Yu-Chueh Hung, W. Yuan, DongHo Cha, K. Geary, and H. R. Fetterman \\ Department of Electrical Engineering, University of California, Los Angeles, California 90095
}

\author{
Soon Mok Ha and Qibing Pei \\ Department of Materials and Science Engineering, University of California, Los Angeles, California 90095
}

Jindong Luo, XingHua Zhou, and Alex K.-Y. Jen

Department of Chemistry, University of Washington, Seattle, Washington 98195

M.-S. Kwon and W. H. Steier

Department of Electrical Engineering, University of Southern California, Los Angeles, California 90089

(Received 27 September 2006; accepted 12 May 2007; published online 14 June 2007)

\begin{abstract}
Metal-slotted optical waveguides (MSOWs) using an electro-optic polymer material have been experimentally demonstrated. The device consists of a three-layered slab waveguide in that the thin metal (gold) film strips are embedded on top of the lower cladding. The optical mode shapes and effective index of the propagation modes of the proposed waveguide structure were calculated using a simplified effective index method and a simulation tool. The fabrication and the device characteristics of a variable optical attenuator and an optical phase modulator based on MSOWs are discussed. () 2007 American Institute of Physics. [DOI: 10.1063/1.2746414]
\end{abstract}

A number of techniques aimed at developing optical wave guided devices have been reported, including reactive ion etching, ultraviolet bleaching, laser writing, poling induced writing, molding/embossing, and metal definition in both passive and active polymers. ${ }^{1,2}$ Recently, based on these fabrication techniques, there have been extensive efforts to produce organic passive and active [electro-optic (EO)] photonic devices, including optical modulators/couplers, ring resonators, digital optical switches, and variable optical attenuators. $^{3-5}$ One of the major reasons to use polymer materials in photonic systems is their exceptional processability $^{6}$ that permits the fabrication of threedimensional optical circuitry, the integration of organic materials with silicon photonics, and with semiconductor verylarge-scale integration electronics by the combination of device concepts and the fabrication techniques mentioned above.

Yamamoto et $a .^{7}$ proposed that an optical waveguide structure of $\mathrm{Al}_{2} \mathrm{O}_{3}$ core $/ \mathrm{SiO}_{2}$ cladding/Si substrate can be a metal film as a partial cladding layer (the so-called metalclad optical strip line). Later, Shelton et al. ${ }^{8}$ investigated metallic gap optical waveguides in GaAs, and $\mathrm{Ma}$ and $\mathrm{Liu}^{9}$ analyzed the optical characteristics of a waveguide structure of $\mathrm{SiO}_{2} / \mathrm{GaAs} / \mathrm{AlGaAs}$ using a simplified effective index method while introducing the term metal-gap optical stripline waveguide. Peng and Oliner ${ }^{10}$ also analyzed the similar structure using a rigorous mode-matching procedure in which the term "optical slot waveguide" was introduced.

At optical frequencies, the dielectric constant $\varepsilon$ of a metal is complex $\varepsilon=\varepsilon^{\prime}+i \varepsilon^{\prime \prime}$, and the propagation constant $\beta$ of a metal-clad dielectric waveguide is also complex, $\beta=\beta^{\prime}+i \beta^{\prime \prime}$, where $\varepsilon$ and $\beta$ are real for dielectric waveguides. This is the main difference between metal-associated dielectric waveguides and dielectric waveguides. ${ }^{11}$ In order to solve the complex eigenvalue equations in the proposed waveguide structure, a simplified approximate technique us-

\footnotetext{
${ }^{a)}$ Electronic mail: kimsku@ee.ucla.edu
}

ing the effective index method was utilized. The index of refraction and propagation loss of the propagation modes of the waveguide were evaluated based on the effective index method and simulation software based on the finite element method.

A schematic cross-sectional view of the proposed dielectric waveguide is shown in Fig. 1. The optical waveguide employs three polymer layers, in which two metallic thin films are placed with a separation $a$ between the two metal strips. We designed the metal-slotted optical waveguides (MSOW) in two ways: (1) MSOW-A has two separated thin $4 \mu \mathrm{m}$ wide metal strip lines [Fig. 1(a)] and (2) MSOW-B has two infinite metal plates [Fig. 1(b)]. The metal separation a between two the metal films is $4 \mu \mathrm{m}$ in both waveguide structures.

The guest material, ${ }^{12}$ AJL8, is doped in a host material, amorphous polycarbonate (APC) (Aldrich Chemical Co.). Recent research reveals that this chromophore has strong nonlinear properties with thermal and photostability. ${ }^{13}$

The confinement of the field in the vertical direction of both waveguide structures is achieved by the layered dielectrics with the core material having higher permittivity than that of the cladding. For the horizontal direction, the field confinement can be understood by studying the difference between the effective permittivities of the center and outer regions. An approximate solution for the MSOW can be found using the effective index method. According to the

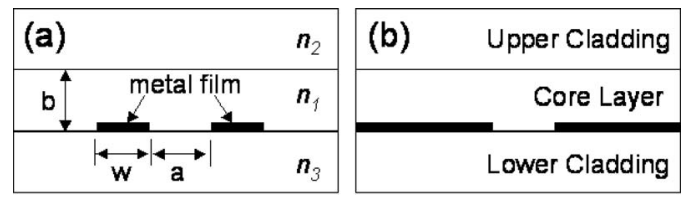

FIG. 1. Proposed device models. (a) MSOW-A with a limited microstrip width, (b) MSOW-B with an infinite microstrip width. With $w=4 \mu \mathrm{m}$, a single mode operation for TM polarization was observed when the metal thicknesses of 80 and $150 \AA$ A thin films are used. Silicon wafer was used for the substrate that is not shown in this figure. 


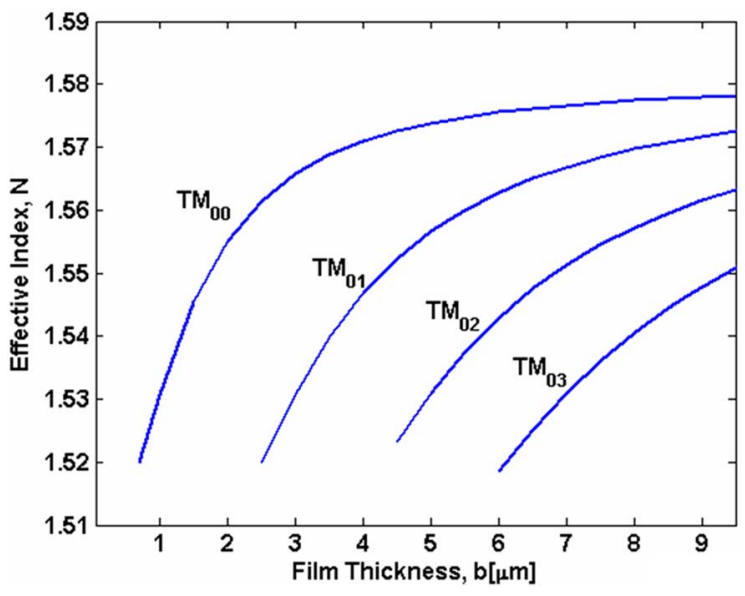

FIG. 2. Effective index $N$ as a function of the gold metal gap, in the case of MSOW-B for TM polarization at $1550 \mathrm{~nm}$. The refractive indices used in this calculation are 1.504 for lower cladding, 1.61 for core layer (AJL8$\mathrm{APC}$ ), and 1.488 for the upper cladding. The core layer was treated as an unpoled EO polymer for this calculation.

effective index method, ${ }^{14}$ we convert a two-dimensional problem into a one-dimensional problem. Therefore, the MSOW was stretched out along the vertical axis, forming two types of regions, namely, the normal slab waveguide and the metal-clad region. The normal slab waveguide and metalclad regions consist of three layer (clad/core/clad) and a four layer (clad/core/metal/clad) structures, respectively. The two one-dimensional slab waveguides can be analyzed in terms of the transverse electric (TE) polarization or the transverse magnetic (TM) polarization modes to find the allowed value of the propagation constant $\beta$ in each region. Once $\beta$ is found, the effective index of the slab is determined through $N_{1}=\beta / k_{0}$ in the normal slab waveguide and $N_{2}=\beta / k_{0}$ in the metal-clad region, where $k_{0}$ is the vacuum wave number of the light being guided. Then the effective index of the whole structure may be determined by solving for the equivalent horizontal slab structure. The dispersion equations ${ }^{9,15,16}$ for the proposed MSOW structure is given by

$$
k_{0} \sqrt{E_{1}-N^{2}} a=m \pi+2 \arctan \left[\frac{\sqrt{\left(N^{2}-E_{2}\right)}}{\sqrt{\left(E_{1}-N^{2}\right)}}\right]
$$

for the TM polarization, where $N$ is the effective index of the mode with the mode number $m=0,1,2,3, \ldots, E_{1}=N_{1}^{2}$ with $N_{1}$ the real effective index of the mode of the slab waveguide (the normal slab waveguide region) and $E_{2}=N_{2}^{2}$ with $N_{2}$ the effective index of the mode of the slab waveguide with the metal layer. The parameter $a$ is the gap between the two metal films and $k_{0}(=2 \pi / \lambda)$ is the wave number in free space. Figure 2 depicts the effective index of the MSOW versus the thickness of the core layer. The number of guided modes increases as the core layer thickness increases. The cutoff of the fundamental mode is about $0.75 \mu \mathrm{m}$ at a $1.55 \mu \mathrm{m}$ wavelength.

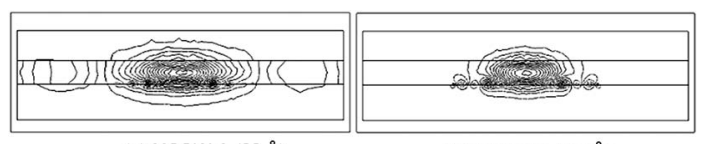

(a) MSOW-A $(80 \AA)$

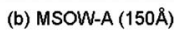

FIG. 3. Device simulation. (a) MSOW-A with a metal thickness of $80 \AA$, (b) MSOW-A with $150 \AA$, respectively $(T M \lambda=1550 \mathrm{~nm})$. The separation $a$ between the two metal films is $4 \mu \mathrm{m}$, and the metal width is $4 \mu \mathrm{m}$.

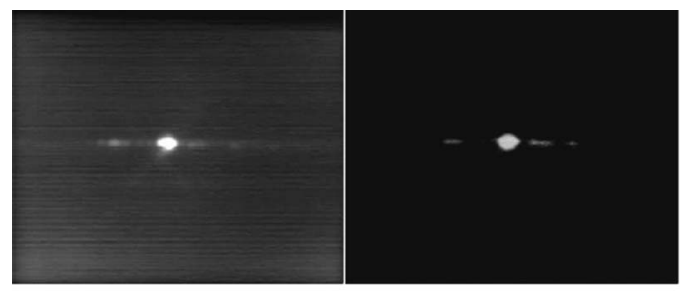

(a) MSOW-A (80Å)

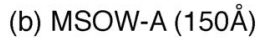

FIG. 4. TM near-field patterns for single mode operated MSOWs. (a) MSOW-A with a metal thickness of $80 \AA$ and (b) MSOW-A with $150 \AA$, respectively.

In order to confirm the guided mode confinement of the single propagating mode, the Helmholtz equation is solved by finite element method (commercial software, FEMLAB) with complex permittivity for certain parts of the structure. The obtained TM mode distributions in MSOW-A are shown in Fig. 3 with two different choices of metal thickness. The permittivity of the gold layer used in this simulation is $-131.94-j 12.65$. $^{17}$ The spacing between the gold layers is $4 \mu \mathrm{m}$ for both MSOW-A and MSOW-B. For MSOW-A [shown in Fig 1(a)], the effective refractive indices of $\mathrm{TM}_{00}$ modes for 80 and $150 \AA$ Au thickness using FEMLAB software are calculated to be $1.582974-j 3.250604 \times 10^{-4}$ and $1.581362-j 8.729443 \times 10^{-4}$, respectively.

The MSOWs are comprised of polymer materials for lower (UV15, Masterbond Co.) and upper (UFC170A, Uray Co.) claddings and core material (AJL8-APC). Gold deposition was performed using an electron-beam evaporator at $0.3 \AA / s$. The layer thicknesses of the fabricated device were lower cladding of $3.0 \mu \mathrm{m}$, core layer of $2.0 \mu \mathrm{m}$, and upper cladding of $2.5 \mu \mathrm{m}$.

Figure 4 shows observed near-field patterns, when TM polarized light at $1550 \mathrm{~nm}$ was launched into the waveguides. The experimental results were in good agreement with the simulated optical power distribution, as shown in Fig. 3. The fabricated waveguides in both MSOW-A and MSOW-B (not shown in Fig. 3) support only a single mode at a $1550 \mathrm{~nm}$, and their optical modes were well confined in the lateral direction and within the core layer.

Figure 5 depicts an application of the MSOW-A for a variable optical attenuator (VOA) that has a multimoded waveguide section underneath a thin film heater. The wave-

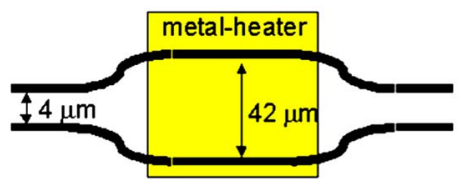

(a) Top view

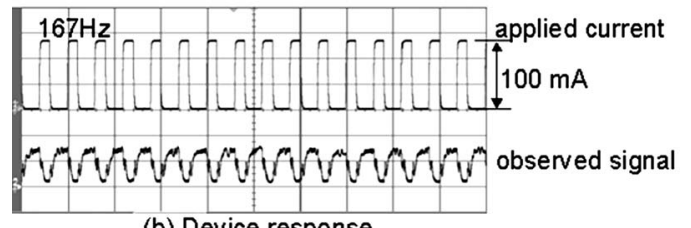

(b) Device response

FIG. 5. Device response of a modified MSOW-A with $150 \AA$, when a square-wave current source of $100 \mathrm{~mA}$ was applied. (a) Top view of the device; (b) the upper trace is the driving signal and the lower trace is the optical signal detected in the device output. VOA oneration was achieved using the thermo-optic effect. 


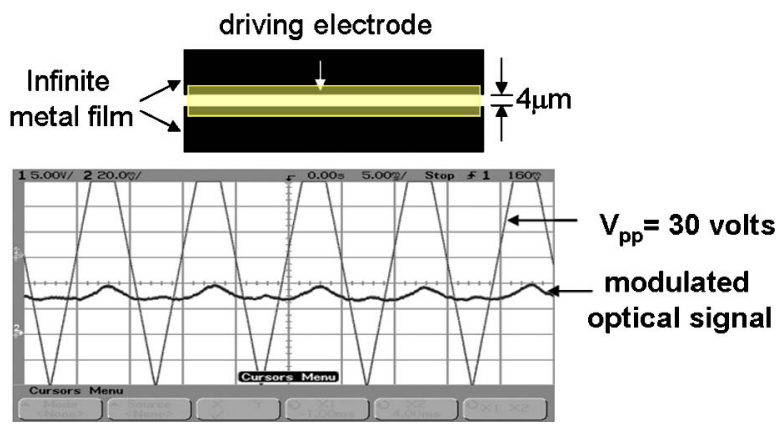

FIG. 6. Low-frequency electro-optic response of MSOW-B with a metal thickness of $150 \AA$ (TM, $\lambda=1550 \mathrm{~nm}$ ). The device length is $2.5 \mathrm{~mm}$.

guide gap between the two metal slots was $42 \mu \mathrm{m}$, and the width of the thin metal film was $4 \mu \mathrm{m}$ for MSOW-B with a $150 \AA$ gold thin film. A $500 \AA \mathrm{Au}$ thin layer drive electrode was defined on top of the upper cladding. A square-wave with a peak current of $100 \mathrm{~mA}$ with a maximum frequency of $167 \mathrm{~Hz}$ from a Keithley 220 programable current source was sent to the electrode and monitored optical output signal using an oscilloscope. The fiber-to-lens loss was measured to be $13 \sim 18 \mathrm{~dB}$ for TM polarization. The total device length was $1.8 \mathrm{~cm}$. Waveguide structures with wider gaps tend to show lower optical propagation loss.

Figure 6 shows the modulation curve of an active EO phase modulator that was made using MSOW-B with a $150 \AA$ gold thin film. The total device length was $5 \mathrm{~mm}$ and electrode poled at $150{ }^{\circ} \mathrm{C}$ of temperature with $400 \mathrm{~V}$ of poling voltage in a nitrogen purged box. The fiber-to-lens optical loss was measured to be $25 \mathrm{~dB}$ in $2.5 \mathrm{~mm}$ for TM polarization. The driving voltage was more than $30 \mathrm{~V}$ in a $2.5 \mathrm{~mm}$ length due to short interaction length.

The optical propagation loss was strongly dependent on the gap and thickness of the metal. The main source of the optical loss in the MSOW is the optical absorption by the thin layer of gold. We believe that the MSOW could exhibit an improved optical propagation loss with a wider metal gap and lower metal thickness. The dominant guiding mode excited in the MSOW was the TE polarization mode and there was a poling induced birefringence in the MSOW due to the preferential alignment of the core chromophore along the planar surface of the wafer. The indices of refraction of the unpoled device were measured to be 1.589 and 1.575 for TE and TM polarizations, respectively. After device poling, the refractive index increases to 1.598 for the TM polarization and decreases to 1.581 for the TE polarization. There was a strong poling induced birefringence for both polarizations.

In this letter, the concept of MSOW in polymers was demonstrated, and its applications for a VOA and an optical phase modulator were discussed. The MSOW could be an alternative way to define an optical waveguide in polymerintegrated optics when the guided waveguide structure parameters such as the metal thickness, the distance between the metal plates, and the waveguide core layer thickness are optimized.

${ }^{1}$ S. K. Kim, K. Geary, W. Yuan, H. R. Fetterman, D. G. Lee, C. Zhang, C. Wang, W. H. Steier, G.-C. Park, S.-J. Gang, and I. Oh, Appl. Phys. Lett. 87, 011107 (2005).

${ }^{2}$ L. Eldada, IEEE J. Sel. Top. Quantum Electron. 6, 54 (2000).

${ }^{3}$ L. Eldada, Opt. Eng. (Bellingham) 40, 1165 (2001).

${ }^{4}$ H. Ma, A. K.-J. Jen, and L. R. Dalton, Adv. Mater. (Weinheim, Ger.) 14, 1339 (2002).

${ }^{5}$ L. R. Dalton, Opt. Eng. (Bellingham) 39, 589 (2000).

${ }^{6}$ L. Dalton, B. Robbin, A. Jen, P. Ried, B. Eichinger, P. Sullivan, A. Akelaitis, D. Bale, M. Haller, J. Luo, S. Lui, Y. Liao, K. Firestone, A. Sago, N. Bhatambrekar, S. Bhattacharjee, J. Sinness, S. Ammond, N. Bucker, R. Snoeberger, M. Lingwood, H. Rommel, J. Amend, S.-H. Jang, A. Chen, and W. H. Steier, Proc. SPIE 5990, 59900C (2005).

${ }^{7}$ Y. Yamamoto, T. Kamiya, and H. Yanai, Appl. Opt. 14, 322 (1975).

${ }^{8}$ J. C. Shelton, F. K. Reinhart, and R. A. Logan, J. Appl. Phys. 50, 6675 (1979).

${ }^{9}$ C. Ma and S. Liu, Opt. Commun. 69, 357 (1989).

${ }^{10}$ S.-T. Peng and A. A. Oliner, IEEE Trans. Microwave Theory Tech. MTT29, 843 (1981).

${ }^{11}$ S. X. She and F. C. Xie, Opt. Commun. 103, 365 (1993).

${ }^{12}$ J. Luo, S. Liu, M. Haller, J.-W. Kang, T.-D. Kim, S.-H. Jang, B. Chen, N. Tucker, H. Li, H. Tang, L. R. Dalton, Y. Liao, B. H. Robinson, and A.-Y. Jen, Proc. SPIE 5351, 36 (2004).

${ }^{13}$ C. Ma and S. Liu, Opt. Quantum Electron. 20, 323 (1988).

${ }^{14}$ C. R. Pollock, Fundamentals of Optoelectronics (Richard D. Irwin, Chicago, 1995), Chap. 8, p. 229.

${ }^{15}$ Y. Suematsu, M. Hakuta, K. Furuya, K. Chiba, and R. Hasumi, Appl. Phys. Lett. 21, 291 (1972).

${ }^{16}$ C. Ma and S. Liu, J. Opt. Soc. Am. A 7, 1577 (1990).

${ }^{17}$ R. Charbonneau, P. Berini, E. Berrolo, and E. Lisicka-Shrez, Opt. Lett. 25, 844 (2000). 